\title{
Improvement analysis of long-span bridges flutter: Messina bridge example
}

\author{
J. Á. Jurado ${ }^{1}$, A. León ${ }^{2}$, F. Nieto ${ }^{2} \&$ S. Hernández ${ }^{2}$ \\ ${ }^{I}$ School of Civil Engineering, University of Coruña, Elviña Campus, \\ La Coruña, Spain \\ ${ }^{2}$ University of Coruña, Elviña Campus, La Coruña, Spain
}

\begin{abstract}
The hybrid methods used in the aeroelastic analysis of flutter for long-span bridges are computational, but they use coefficients and functions obtained experimentally in a wind tunnel. The experimental testings are carried out with a deck sectional model and there are two types: an aerodynamic testing for obtaining the aerodynamic coefficients such as drag, lift and moment, and an aeroelastic testing for obtaining the flutter derivatives, that the model oscillates under free vibration suspended by springs. In this paper, some improvements were achieved in the experimental phase as well as in the computational phase of the method. The influence of variation of the aerodynamic coefficients were studied with Reynold's number; different sets of springs were used to include a wide range of reduced velocities that the flutter derivatives depend on; the influence of deformation that the static wind load produces at the angle of attack along the bridge span was studied; finally this angle of attack was taken into account to determine the flutter derivatives used at each part of the deck. These improvements were applied to the sectional and computational models of the future Messina Strait Bridge in Italy.
\end{abstract}

Keywords: aeroelasticity, long-span bridges, flutter, sectional tests.

\section{Introduction}

The flutter condition on long-span bridges is critical during the design of these structures. To avoid experimental tests of completed bridge models in large wind tunnels that are complicated and expensive, it is necessary to use a hybrid method which is computational based but needs experimental parameters. 
Sectional models of the deck are initially tested in an aerodynamic wind tunnel of smaller dimensions to obtain the flutter derivatives. These coefficients are then used in the computational analysis of the aeroelastic behaviour of the completed bridge.

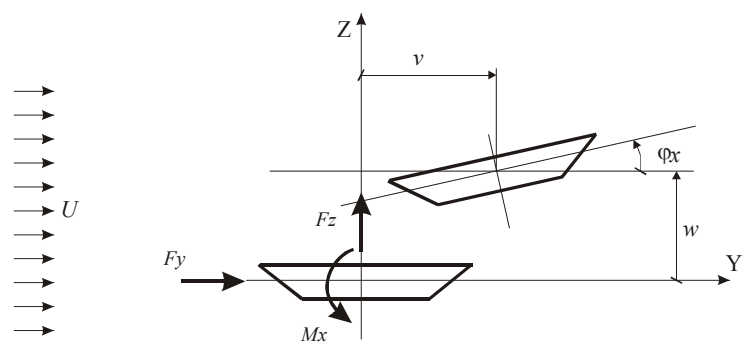

Figure 1: $\quad$ Forces and displacements of a sectional model.

Figure 1 shows the three forces acting on a deck. According to Simiu and Scanlan [1] formulation, these actions are linearized as functions of the displacements and velocities of the system for vertical $w$, lateral $v$ and torsional rotation $\varphi_{x}$ degrees of freedom. As it was explained in Jurado and Hernández (2000), the flutter condition is obtained by the computational solving of a nonlinear eigen-problem which comes from the dynamic balance equation for the deck.

$$
\mathbf{M u ̈}+\mathbf{C} \dot{\mathbf{u}}+\mathbf{K u}=\mathbf{f}_{a}=\mathbf{K}_{a} \mathbf{u}+\mathbf{C}_{a} \dot{\mathbf{u}}
$$

$\mathbf{M}, \mathbf{C}$ and $\mathbf{K}$ are respectively the mass, damping and stiffness structural matrices. $\mathbf{f}_{a}$ is the aeroelastic forces vector which can be written assembling aeroelastic forces $F_{y} F_{z} M_{x}$ along the deck as a stiffness $\mathbf{K}_{a}$ and damping $\mathbf{C}_{a}$ aeroelastic matrices multiplied by the displacements $\mathbf{u}$ and velocities $\dot{\mathbf{u}}$ vectors. The expressions of these matrices are

$$
\mathbf{C}_{a}=\frac{1}{2} \rho U^{2} K B l \cdot\left(\begin{array}{ccc}
P_{1}^{*} & -P_{5}^{*} & -B P_{2}^{*} \\
-H_{5}^{*} & H_{1}^{*} & B H_{2}^{*} \\
-B A_{5}^{*} & B A_{1}^{*} & B^{2} A_{2}^{*}
\end{array}\right) \quad \mathbf{K}_{a}=\frac{1}{2} \rho U^{2} K^{2} l \cdot\left(\begin{array}{ccc}
P_{4}^{*} & -P_{6}^{*} & -B P_{3}^{*} \\
-H_{6}^{*} & H_{4}^{*} & B H_{3}^{*} \\
-B A_{6}^{*} & B A_{4}^{*} & B^{2} A_{3}^{*}
\end{array}\right)
$$

where $B$ is the deck width, $\rho$ is the air density, $U$ is the mean wind speed, $K=$ $B \omega / U$ is the reduced frequency with $\omega$ the frequency of the response and $H_{i}{ }_{i}(K)$, $P^{*}{ }_{i}(K), A^{*}{ }_{i}(K) \mathrm{i}=1 . .6$ are the flutter derivatives which are functions of $K$.

In the following sections, the improvements made in the sectional bridge deck testings are explained as well as the improvements in the computational calculation of the critical flutter velocity for the entire bridge structure. Those advances are applied to the future Messina strait bridge model, between Sicily and the Italian peninsula. 


\section{Aerodynamic sectional testing of the Messina Bridge}

In order to carry out the static analysis of the wind load, we need to obtain previously the aerodynamic coefficients of the deck cross section in function of the angle of attack. The aerodynamic coefficients are obtained by carrying out a testing of a fixed deck sectional model measuring inside the wind tunnel drag force $D_{s}$, lift $L_{s}$ and moment $M_{s}$ that exerts air flow over the model. See figure 2.

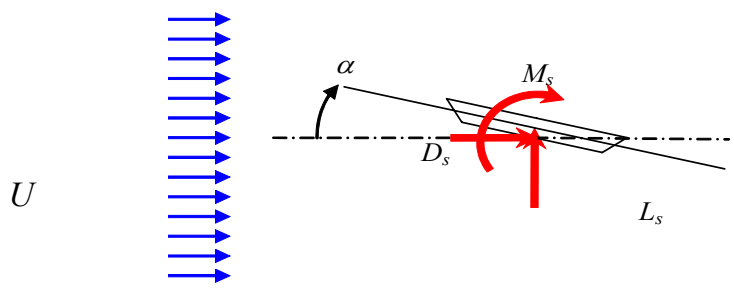

Figure 2: Aerodynamic forces on a sectional model.

First of all, a scale bridge deck sectional model is built, whose shape should be as similar to the prototype as possible. Figure 3 shows a sectional model of the Messina Bridge. The mass does not need to be scaled to the original bridge since the displacements are constrained and there are no inertial forces. We simply search for simulating the real boundary conditions that determine the air flow around the deck.

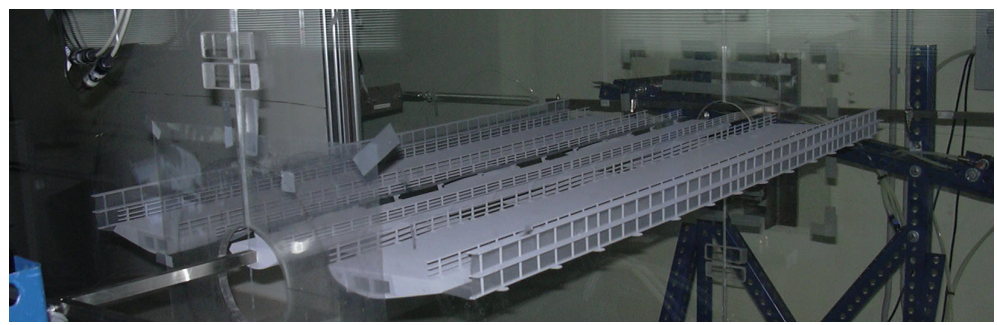

Figure 3: $\quad$ Sectional deck model of the Messina Strait Bridge.

An important problem for obtaining the aerodynamic coefficients is its dependence on Reynolds number $(\operatorname{Re}=\rho U B / \mu$; $\rho$ : air density, m: air viscosity; $U$ : wind velocity, $B$ : deck width), see Son and Hanratty [2]. As a consequence the aerodynamic forces and the aerodynamic coefficients change noticeably when the wind velocity increases as shown in Figure 2. From a certain wind velocity value, the coefficient values are quite stable. It is essential to determine the correct air flow velocity in the wind tunnel to obtain the aerodynamic forces. This should be carried out by trying with different angles of attack as shown in Figure 4 for the Messina Strait Bridge. In the graph, it can be observed that with Reynold's number over 400000 , the values do not vary noticeably. Once the 
velocity is determined, $11 \mathrm{~m} / \mathrm{s}$ for the Messina example which corresponds to Re $=460000$, the testings were carried out varying the angle of attack to obtain the aerodynamic coefficient graphs like the one shown in figure 5.

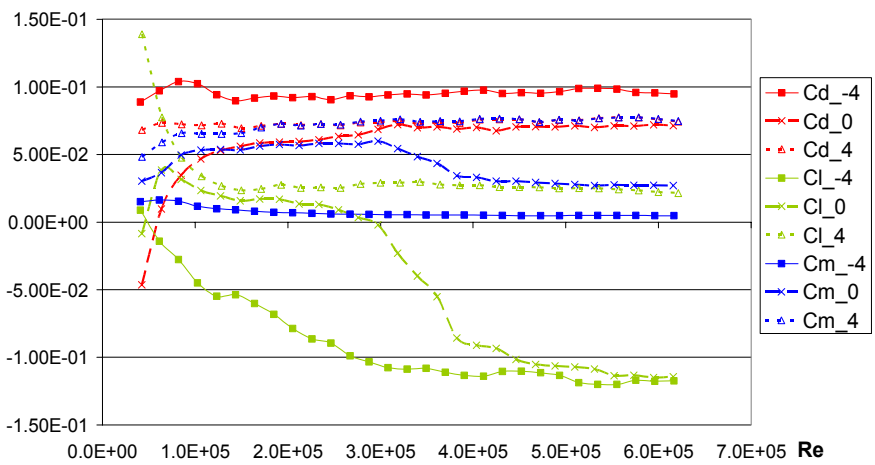

Figure 4: Variation of the aerodynamic coefficients of the Messina Bridge deck for different angles of attack in function of Reynold's number.

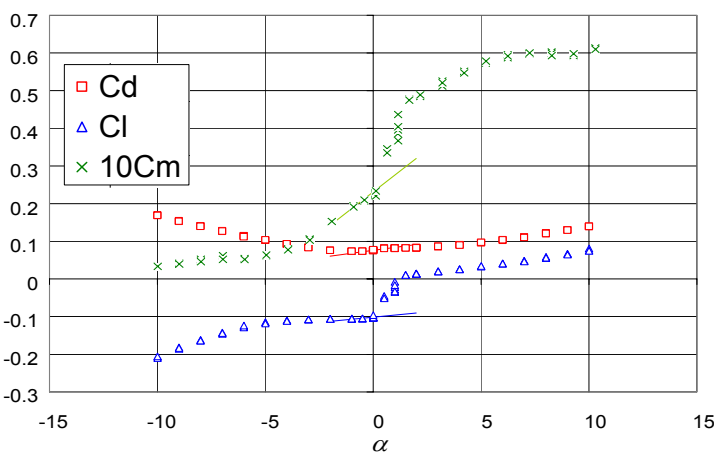

Figure 5: Aerodynamic coefficients of the Messina Bridge deck.

\section{Aeroelastic sectional testing of the Messina Bridge}

The aeroelastic sectional testings under free vibration consist of sustaining the sectional model by springs and make them freely oscillate with and without air flow inside the wind tunnel. A detailed explanation of this procedure is found in Jurado et al [3]. From the displacements of the model, we can calculate the stiffness properties and damping, for example with the MITD (Modified Ibrahim and Mikulcik [4] Time Domain Method), and from the variation of those properties as the wind velocity varies, we can obtain the flutter functions. See Sarkar et al [5].

A sectional model requires less similarity conditions than a reduced complete bridge model. The most important thing is to maintain the geometric similarity. It is recommended that the length of the model is three times its width in order to 
be considered two-dimensional. Scales less than 1/100 should not be used. The mass and inertia values are not very important for the testing even though it is recommendable that they are small enough for not entering errors in measuring the wind forces. Therefore there is no need to consider a scale of masses since we only try to quantify the wind action in function of the oscillatory movements of the deck. The model is elastically sustained using eight to twelve springs: four or eight vertical and four horizontal ones (Figure 6).
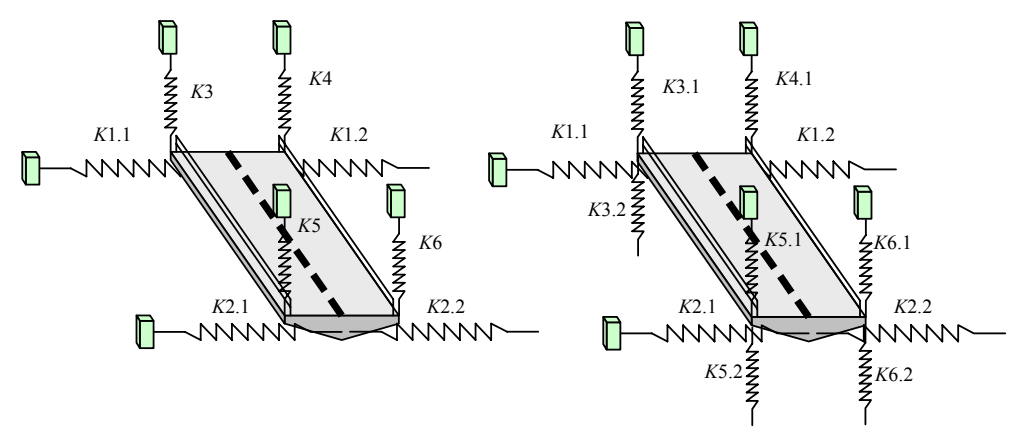

Figure 6: Sustentation of the sectional model with eight or twelve springs.

The stiffness of the springs determines the vibration frequencies $(2 \pi f=\omega)$ of the system that together with the wind velocity in the tunnel, $V$ and the model width, $B$, determines the range of reduced velocities, $V^{*}$, in order to be able to obtain flutter functions.

$$
V^{*}=V / f B=2 \pi V / \omega B=2 \pi / K
$$

For example, eighteen flutter functions of the deck model of the Messina strait bridge were obtained considering three aerodynamic appendages at the wind shields. Testings were carried out varying the angle of attack between $-3^{\circ}, 0^{\circ}$ and $+3^{\circ}$. Four types of sustaining the model with different springs were used in order to vary the natural frequencies of the model and include a wider range of reduced velocities. For the first three testings, the model was allowed to have vertical and horizontal displacements and rotation, while in the fourth one, it was only allowed to rotate. The model for the first testing was sustained with twelve springs, and for the second and the third testings it was sustained with eight springs. For the fourth testing, the model was not allowed to move vertically or horizontally with two bars in each side of the model. The mass of the model is $7.47 \mathrm{~kg}$ and its torsional moment of inertia is $0.35 \mathrm{~kg} \cdot \mathrm{m}^{2}$, and the natural frequencies of the system for each sustaining type are the followings.

The testings were carried out for wind velocities between 6 and $20 \mathrm{~m} / \mathrm{s}$. The minimum and maximum reduced velocities with which flutter functions can be obtained are shown in Table 2. With the testings of three degrees of freedom, 18 flutter functions are obtained simultaneously, while with the testing of only rotational freedom, we can only identify the flutter functions, $A_{2}{ }^{*}$ and $A_{3}{ }^{*}$. Figure 7 shows the obtained flutter functions. 
16 The Art of Resisting Extreme Natural Forces

Table 1: $\quad$ Natural frequencies of the aeroelastic testings.

\begin{tabular}{|c|ccc|}
\hline & $f v(\mathrm{~Hz})$ & $f w(\mathrm{~Hz})$ & $f \varphi_{\mathrm{x}}(\mathrm{Hz})$ \\
\hline Test 1 & 2.2 & 2.9 & 6.8 \\
Test 2 & 2.8 & 1.7 & 3.5 \\
Test 3 & 1.3 & 1.4 & 2.0 \\
Test 4 & 0 & 0 & 1.14 \\
\hline
\end{tabular}

Table 2: Range of reduced velocities for obtaining different flutter functions.

\begin{tabular}{|c|cc|c|c|c|c|}
\hline & \multicolumn{2}{|c|}{$\left(A_{5}{ }^{*}, A_{6}{ }^{*}, H_{5}{ }^{*}\right.}$, & \multicolumn{2}{c|}{$\left(A_{1}^{*}, A_{4}{ }^{*}, H_{1}{ }^{*}\right.}$, & \multicolumn{2}{c|}{$\left(A_{2}{ }^{*}, A_{3}{ }^{*}, H_{2}{ }^{*}\right.}$, \\
& $\left.H_{6}{ }^{*}, P_{1}{ }^{*}, P_{4}{ }^{*}\right)$ & \multicolumn{2}{c|}{$\left.H_{4}{ }^{*}, P_{5}{ }^{*}, P_{6}{ }^{*}\right)$} & \multicolumn{2}{|c|}{$\left.H_{3}{ }^{*}, P_{2}{ }^{*}, P_{3}{ }^{*}\right)$} \\
\cline { 2 - 7 } & $V^{*}\left(f_{v}\right) \min$ & $V^{*}\left(f_{v}\right) \max$ & $V^{*}\left(f_{w}\right) \min$ & $V^{*}\left(f_{w}\right) \max$ & $V^{*}\left(f_{\varphi x}\right)$ & $V^{*}\left(f_{\varphi x}\right)$ \\
\hline Test 1 & 4.521 & 15.07 & 3.40 & 11.34 & 1.45 & 4.85 \\
Test 2 & 3.54 & 11.79 & 5.82 & 19.41 & 2.82 & 9.39 \\
Test 3 & 7.62 & 25.38 & 7.22 & 24.09 & 4.90 & 16.34 \\
Test 4 & - & - & - & - & 8.68 & 28.95 \\
\hline
\end{tabular}
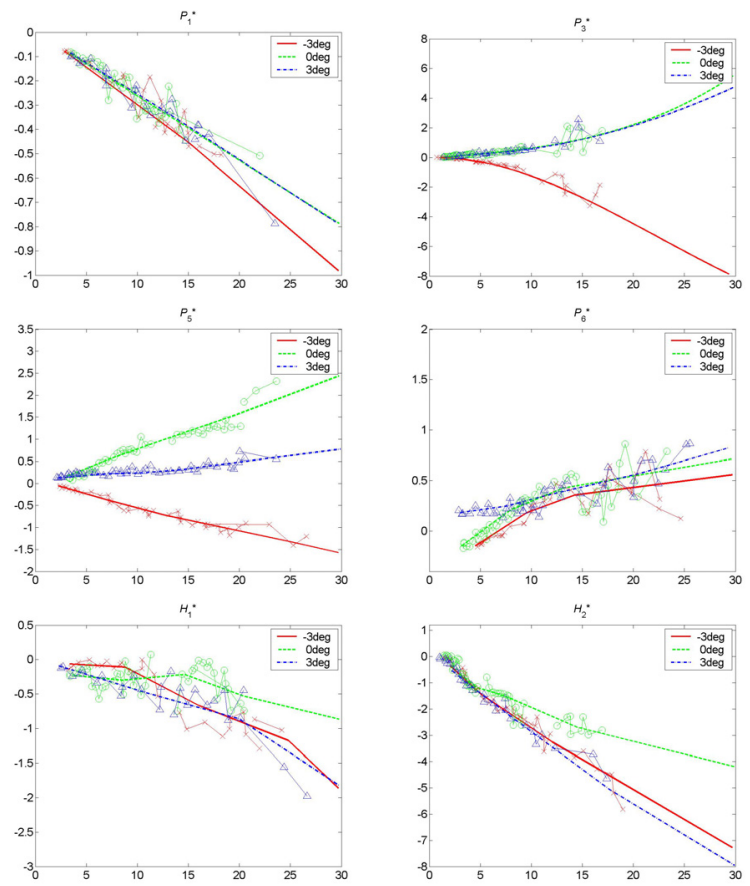

Figure 7: Same flutter derivatives of the deck of the Messina bridge in function of the reduced velocity $V^{*}=2 \pi / K$ (x axis). 

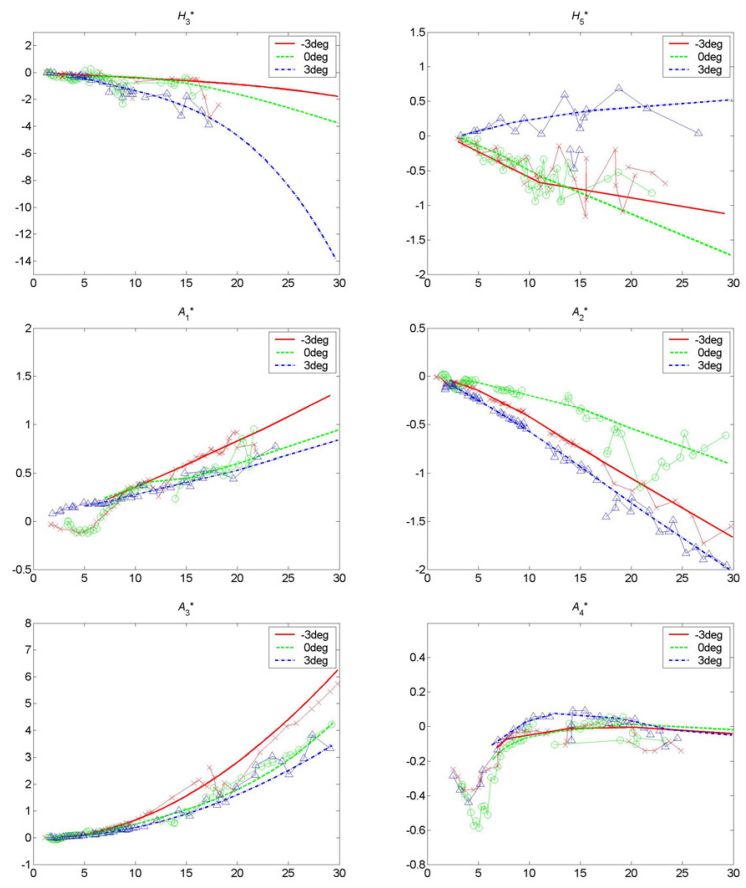

Figure 7: Continued.

\section{Influence of the static deformation on the angle of attack}

A non-linearity that normally not taken into account for wind load analyses of long-span bridges is the variation of angle of attack due to deck deformation. Rotation about the axis of the deck can vary some degrees along the deck; for example, $0.6^{\circ}$ for the Messina Bridge [6] at the centre span, and $3^{\circ}$ for the Akashi Bridge. The deck rotation causes a change in the angles of attack, which affects the aerodynamic coefficient and the flutter coefficient values. Calculation of the static solution of the wind is carried out by resolving the equation system that relates the forces on the structure and the displacements. This system can be expressed in matrix form as:

$$
\mathbf{K}(\mathbf{u}) \cdot \mathbf{u}=\mathbf{f}[\boldsymbol{\alpha}(\mathbf{u})]
$$

where not only the stiffness matrix, $\mathbf{K}$ depends on the displacements, $\mathbf{u}$, but also the wind loads, $\mathbf{f}$ that vary with the angle of attack, $\alpha$ throughout the deck and therefore also depend on $\mathbf{u}$. If this dependency is not taken into account, (4) is converted in a linear problem in which the stiffness matrix and the wind force are used for the initial positions of the nodes with null angle of attack. 
An approximation of the problem (4) consists of considering the deck deformation in the wind forces.

$$
\mathbf{K} \cdot \mathbf{u}=\mathbf{f}[\boldsymbol{\alpha}(\mathbf{u})]
$$

In this approximation, the influence of the wind forces on the structural stiffness is not considered. The problem is resolved by iterations. In each step, new forces that depend on the obtained displacements in the previous step are defined. The process finishes when the obtained displacements converge, that is to say they do not change too much between two iterations. This method was applied to the static deformation of the Messina Bridge observing noticeable differences in the deck displacements for the wind velocity over $50 \mathrm{~m} / \mathrm{s}$ as shown in Figure 8 .
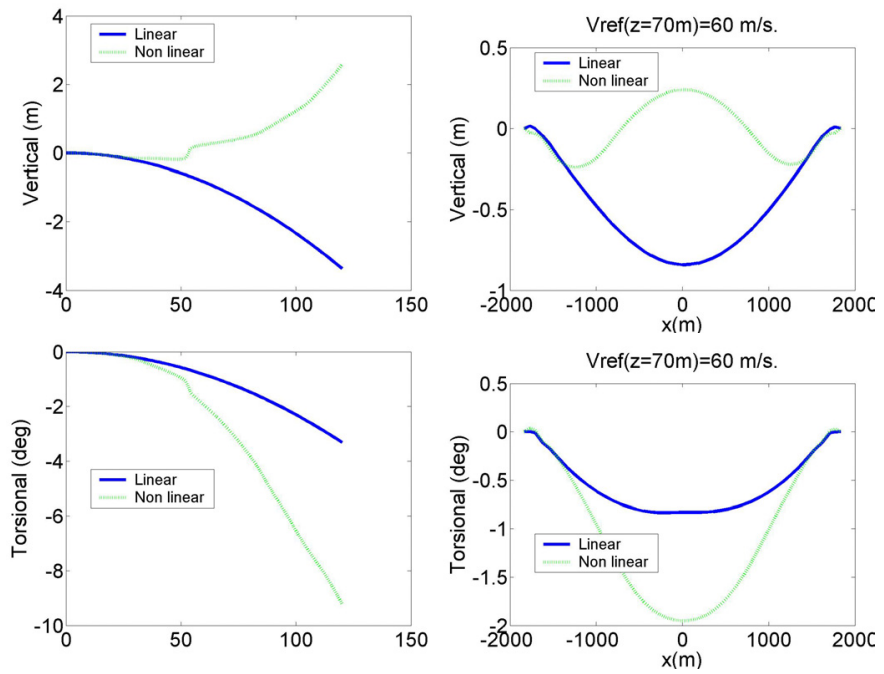

Figure 8: Deck displacements of the Messina Bridge at increasing wind velocity.

\section{Flutter analysis of Messina Bridge}

In the present work, a coherent matrix formulation has been used for hybrid flutter analysis. Jurado and Hernandez [7] explain this formulation stems from the equation (1). Through modal analysis it is possible to approximate the deck displacements by means of a linear combination of the most significant mode shapes. Assembling them in columns into the modal matrix $\Phi$, the displacement vector can be expressed as $\mathbf{u}=\mathbf{\Phi q}$. Each element of the vector $\mathbf{q}$ represents the participation of each mode shape in the displacement vector $\mathbf{u}$. Premultiplying (1) by $\Phi^{T}$ it becomes

$$
\mathbf{I} \ddot{\mathbf{q}}+\mathbf{C}_{R} \dot{\mathbf{q}}+\mathbf{K}_{R} \mathbf{q}=\mathbf{0}
$$


where $\mathbf{C}_{R}=\Phi^{\mathrm{T}}\left(\mathbf{C}-\mathbf{C}_{a}\right) \Phi, \mathbf{K}_{R}=\Phi^{\mathrm{T}}\left(\mathbf{K}-\mathbf{K}_{a}\right) \tilde{\Phi}$ and $\boldsymbol{\Phi}^{T} \mathbf{M} \boldsymbol{\Phi}=\mathbf{I}$ Using mass normalized modes. Knowing that the solution of this equation has the form $\mathbf{q}(t)$ $=\mathbf{w} e^{\mu t}$, becomes

$$
\left(\mu^{2} \mathbf{I} \mathbf{w}+\mu \mathbf{C}_{R} \mathbf{w}+\mathbf{K}_{R} \mathbf{w}\right) e^{\mu t}=\mathbf{0}
$$

which can be transformed into an eigenvalue problem by adding the identity $\mu \mathbf{I} \mathbf{w}+\mu \mathbf{I} \mathbf{w}=\mathbf{0}$ :

$$
\left[\mu\left(\begin{array}{cc}
\mathbf{I} & \mathbf{0} \\
\mathbf{0} & \mathbf{I}
\end{array}\right)\left(\begin{array}{c}
\mu \mathbf{w} \\
\mathbf{w}
\end{array}\right)+\left(\begin{array}{cc}
\mathbf{C}_{R} & \mathbf{K}_{R} \\
-\mathbf{I} & \mathbf{0}
\end{array}\right)\left(\begin{array}{c}
\mu \mathbf{w} \\
\mathbf{w}
\end{array}\right)\right] e^{\mu t}=\mathbf{0}
$$

or in short

$$
(\mathbf{A}-\mu \mathbf{I}) \mathbf{w}_{\mu} e^{\mu t}=\mathbf{0}
$$

The imaginary part of the eigenvalues $\mu$ counts on the frequency $\omega$, while the real part of the eigenvalues is associated with the damping ratio $\xi$. The condition of flutter corresponds to the lowest wind speed $U_{f}$ which gives one eigenvalue with vanished real part. However, the problem (9) is non-linear because the matrix $\mathbf{A}$ assembles the aeroelastic matrices $\mathbf{K}_{a}$ and $\mathbf{C}_{a}$. These matrices contain the flutter derivatives, which are functions of the reduced frequency $K=B \omega / \bar{U}$, and the frequency for each eigenvalue $\omega$ remains unknown until the problem has been solved.
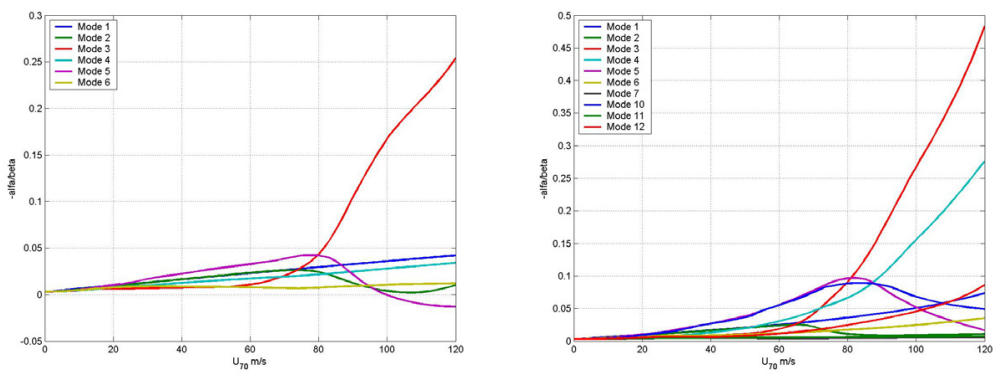

Figure 9: Relation between the real and imaginary part of the eigenvalues in the flutter analysis not considering (left $U_{f}=100 \mathrm{~m} / \mathrm{s}$ ) and considering (right $U_{f}>120 \mathrm{~m} / \mathrm{s}$ ) the variation of angle of attack.

An improvement of the bridge flutter analysis consists of taking into account the dependency of the flutter derivatives on the angle of attack at each point of the deck due to the static deformation, and its calculation is explained in the previous section. Figure 9 shows graphs with the evolution of the proper values of the problem (9) for increasing wind velocities with or without varying the angle of attack. It is observed that the critical flutter velocity increases favourably when this effect is considered. 


\section{Conclusions}

The wind velocity used for the aerodynamic sectional testing in a wind tunnel should be chosen by previously studying the variation of aerodynamic forces in unction of Reynold's number.

If various sets of springs are used in the aeroelastic sectional testings, a bigger range of reduced velocities can be included in obtaining the flutter derivatives.

The static deformation due to the wind load affects considerably the angle of attack at high wind velocities close to the flutter.

The critical flutter velocity also varies noticeably if the variation of angle of attack along the bridge span is taken into account. The flutter derivatives should be chosen according to this dependency.

\section{Acknowledgement}

This research has been funded by the Galician Council of Innovation, Trade and Industry under project PGIDIT04CCP118002PR.

\section{References}

[1] Simiu E., Scanlan R. H., (1996) Wind Effect on Structures, Wiley N. Y.

[2] Son J. S., Hanratty T. J., (1969) Numerical solution for the flow around a cylinder at Reynolds number of 40, 200, 500. J. of Fluid Mech. Vol. 35 pp: 369-386.

[3] Jurado J. Á., León A., Hernández S. (2005) Wind Tunnel Control Software for Identification of Flutter Derivatives on Bridge Sectional Tests. EACWE 4. $4^{\circ}$ European and African Congress in Wind Eng. Prague, Check Republic.

[4] Ibrahim S.R., Mikulcik E. C. (1977) A Method for the Direct Identification of Vibration Parameters from the Free Response, The Shock and Vibration Bulletin, bulletin 47, Part 4.

[5] Sarkar P. P., Jones N. P., Scanlan R. H. (1992) System identification for estimation of flutter derivatives, Journal of Wind Engineering and Industrial Aerodynamics 41-44 1243-1254.

[6] Stretto di Messina S.p.A. (2004), Specifiche tecniche per il progetto definitivo e il progetto esecutivo dell'opera di attraversametnto. Requisiti e linee guida per lo sviluppo della progettazione. GCG.F.05.03.

[7] Jurado, J. Á., Hernandez S. (2004) Sensitivity analysis of bridge flutter with respect to mechanical parameters of the deck. Structural and Multidisciplinary Optimization Vol. 27, N 4. June, 2004. 